\title{
Vulnerability to Reentry in a 3D Regionally Ischemic Ventricular Slab Preparation: a Simulation Study
}

\author{
E Heidenreich ${ }^{1}$, L Romero ${ }^{2}$, JF Rodríguez ${ }^{1}$, B Trénor ${ }^{2}$, \\ JM Ferrero Jr² ${ }^{2}$ S Sáiz ${ }^{2}$, M Doblaré ${ }^{1}$ \\ ${ }^{1}$ I3A-Universidad de Zaragoza, Zaragoza, Spain \\ ${ }^{2}$ Universidad Politécnica de Valencia, Valencia, Spain
}

\begin{abstract}
Ventricular tachycardia and ventricular fibrillation are known to be two types of cardiac arrhythmias that usually take place during acute ischemia and frequently lead to sudden death. In this work, we have studied the different patterns of activation displayed in a virtual ventricular slab preparation after premature stimulation during acute ischemia. Furthermore, we also have analyzed the vulnerable window $(V W)$ under such conditions. Influence of the tissue structure and morphology of the ischemic zone have also been considered. For a centered ischemic zone, eight shaped reentry was originated at the mid plane of the slab and the VW was found to be almost the same as for the $2 D$ simulations. Eight shaped reentry were formed in the epicardial surface as the morphology of the ischemic zone changed (the centre of the ischemic zone was moved toward the epicardial surface). These changes also caused a reduction in the VW of a $24 \%$ as compared with the centered ischemic zone.
\end{abstract}

\section{Introduction}

Ventricular tachycardia and fibrillation are among the major cause of sudden cardiac death [1]. Even though these arrhythmias arise from different clinical conditions, ischemic heart disease is the foremost perpetrator among them. Occlusion of the coronary artery, which causes ischemia, is followed by profound metabolic changes in the intracellular and extracellular space of the cardiac tissue. This metabolic changes are mainly hypoxia, increased concentrations of the extracellular $\mathrm{K}^{+}$ (hyperkalemia), increased concentrations of intracellular $\mathrm{Na}^{+}$, and $\mathrm{Ca}^{2+}$, decreased concentration of extracellular $\mathrm{Na}^{+}$, decrease of intracellular ATP, and acidosis [2,3]. In addition, the impact of ischemia in the myocardium is characterized with a high degree of heterogeneity. Due to diffusion of ions and metabolites, the core of the tissue suffering from the lack of blood (the central ischemic zone, $(\mathrm{CZ})$ is surrounded by a border zone (BZ) which comprises changes in electrophysiological properties between the healthy and ischemic regions $[4,5,6]$. These heterogeneities are produced not only intramurally, but also transmurally, in the depth of the ventricular wall.

From an electrophysiological point of view, these changes imply alterations in action potential configurations, excitability, conduction velocities, refractive period among others, which enormously favor reentrant activity, and therefore arrhythmias and fibrillation $[7,8,9]$.

Over the last years, mathematical modeling and computer simulations have been a useful tool in analyzing electrophysiological phenomena. In this particular, one of the major contributions of computer electrophysiology has been in understanding important relations between electrophysiological parameters [3]. For the ischemic heart, computer models have allowed to address the role of ischemic abnormalities in cardiac electrophysiological behavior [9]. However, most of these simulations have been restricted to $2 \mathrm{D}[8,9]$ simulations or $3 \mathrm{D}$ simulations of total ischemic heart [10]. In this research work we study the patterns of reentry in a 3D virtual tissue subjected to acute regional ischemia. The electrical activity of the cells is described using a second generation model of ventricular action potential. The effect of the cardiac fiber architecture and location of the ischemic zone on the vulnerable window of the tissue is studied.

\section{Methods}

\subsection{Mathematical model}

The tissue has been considered as a continuum, with its electrophysiological behavior responding according to the mono-domain model [11]

$$
\nabla \cdot\left(\bar{\sigma}_{i} \nabla V\right)=\chi C_{\mathrm{m}} \frac{\partial V}{\partial t}+\chi q_{\mathrm{ion}}+\chi q_{\mathrm{stm}},
$$

where $V$ is the transmembrane potential, $\bar{\sigma}_{\mathrm{i}}$ is the anisotropic conductivity tensor, $C_{\mathrm{m}}$ the membrane capacitance, $\chi$ the volume to surface ratio of the cell, 
$q_{\text {ion, }}$ the ionic current, and $q_{\text {stm }}$, a stimulus current. Equation (1) is subjected to the zero flux boundary conditions

$$
\mathbf{n} \cdot\left(\bar{\sigma}_{i} \nabla V\right)=0 .
$$

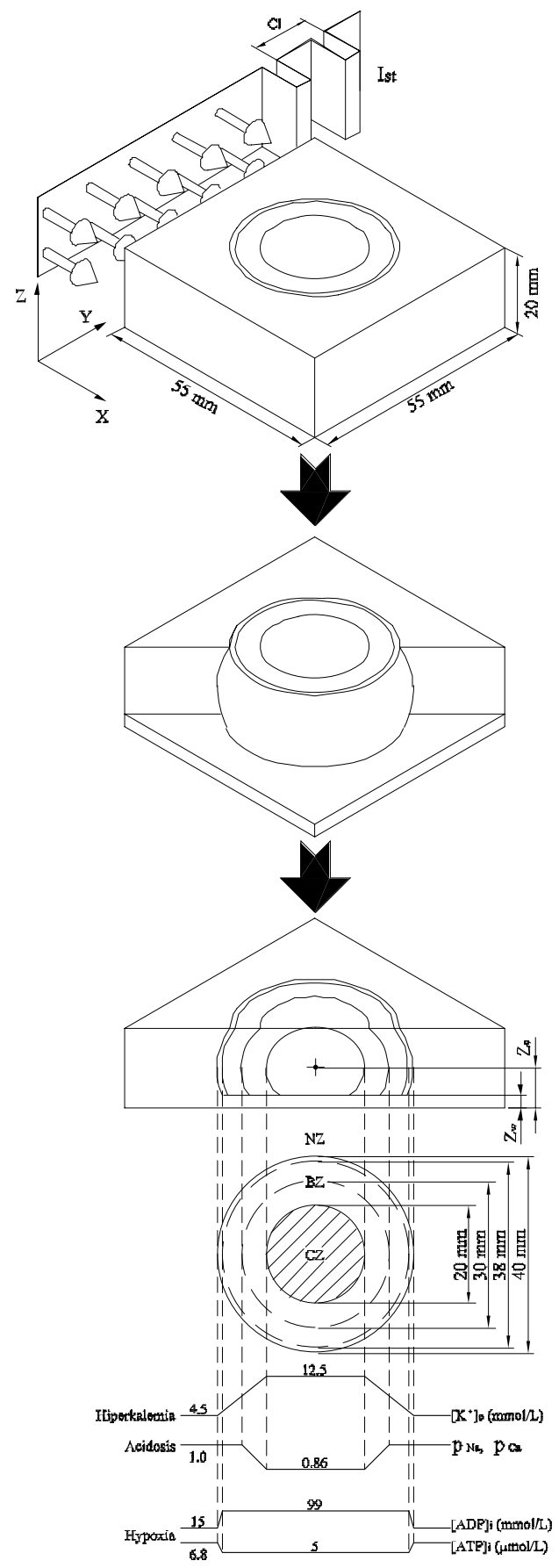

Figure 1. 3D representation of virtual ischemic zones, with the central ischemic zone (CZ), border zone (BZ) and normal zone (NZ).

An important aspect in solving (1) and (2) is the formulation for the ionic currents given in the $q_{\text {ion. A }}$ number of models exist of varying degree of detail of cell physiology. Since we were interested in studying the patterns of excitation in acute ischemia, and the ionic mechanisms responsible of them, a comprehensive model describing the action potential was required. In this research, a modified version of the Luo-Rudy phase two model $[12,13]$ was used. This model describes with great electrophysiological detail the ionic currents responsible for the ventricular action potential, including thirteen transmembrane currents, $\mathrm{Ca}^{2+}$ buffering, internal $\mathrm{Ca}^{2+}$ induced $\mathrm{Ca}^{2+}$ fluxes, as well as a description of intracellular $\mathrm{Ca}^{2+}, \mathrm{K}^{+}$, and $\mathrm{Na}^{+}$ionic concentrations. The model comprises a stiff nonlinear system of ODEs involving the action potential, voltage dependent gating variables, and intracellular ionic concentrations with a total of 30 state variables and time constants ranging from 0.1 to $500 \mathrm{~ms}$.

\subsection{Model of acute ischemia}

Figure 1 shows the electrophysiological structure of the virtual tissue, with the different zones created by acute regional ischemia. The size of each zone as well as the value of the ischemic parameters and the spatial gradients has been adapted from experimental data [5]. The configuration shown in Figure 1 corresponds to 10 minutes form the onset of ischemia. The tissue comprises a normal zone (NZ), a border zone (BZ) and a central zone $(\mathrm{CZ})$. It also shows the washed area in the endocardium.

Hipercalemia was modeled by elevating the concentration of $\left[\mathrm{K}^{+}\right] \mathrm{O}$ from $4.5 \mathrm{mmol} / \mathrm{L}$ in the $\mathrm{NZ}$ to $12.5 \mathrm{mmol} / \mathrm{L}$ [14] in the CZ, with a transition zone of 10 $\mathrm{mm}$ (coinciding with the border zone) in which the $\left[\mathrm{K}^{+}\right] \mathrm{O}$ concentration varies linearly [5]. The effect of acidosis was taken into account by reducing the conductance for the inward $I_{\mathrm{Na}}$ current, and the $\mathrm{Ca}^{2}+$ current through the $L$ type channels, $I_{\mathrm{Ca}, \mathrm{L}}$, by a factor of 0.8625 in the $\mathrm{CZ}$ and 1.0 in the NZ $[15,16]$. The transition was taken as linear, with the size of the transition zone inferred from data from [5]. Hipoxia, which causes a reduction of intracellular ATP and increases free intracellular ADP, leads to the activation of ATP sensitive $\mathrm{K}^{+}$currents (IK,ATP). The model proposed by [8] was used for modeling $I_{\mathrm{K}, \mathrm{ATP}}$ current. The model considers the dependency of $I_{\mathrm{K}, \mathrm{ATP}}$ on $\mathrm{Mg}^{+}$, intracellular ATP and $\mathrm{ADP}$, and $\left[\mathrm{K}^{+}\right] \mathrm{O}$, as well as the voltage dependence of the channel. The values of intracellular ATP and ADP shown in Figure 1 were taken from [17], and the size of the border zone $(1 \mathrm{~mm}$ on the normal side of the BZ) defined according to [18]. 


\subsection{Numerical model}

A $3 \mathrm{D}$ virtual tissue simulating a $55 \mathrm{~mm} \times 55 \mathrm{~mm} \times$ $20 \mathrm{~mm}$ slab of ventricular myocardium subjected to regional ischemia was considered (see Figure 1). Orientation of ventricular muscle fibers varied linearly from $-60^{\circ}$ in the epicardial surface to $+60^{\circ}$ in the endocardial surface. The imbrication angle of the fibers was also accounted for by linearly varying the imbrication angle from $0^{\circ}$, in the epicardium, up to $10^{\circ}$ in the endocardium. A NZ $2 \mathrm{~mm}$ thick was considered in the endocardium to account for the unaffected tissue in contact with interventricular blood. Figure 2 shows the computational domain depicting the ischemic zone and the orientation of the fibers across the myocardium.

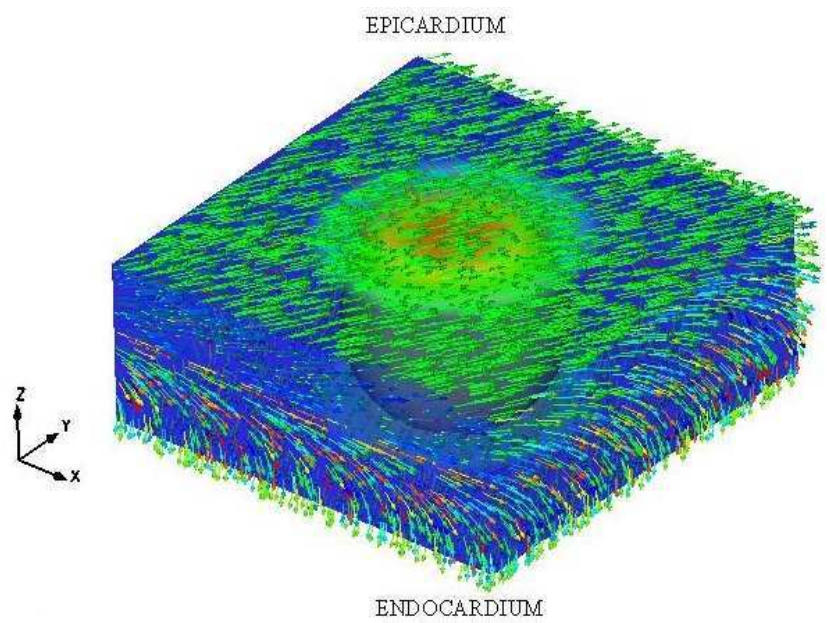

Figure 2. Computational domain used for the simulations. The figure detail the ischemic zone and the direction of the muscular fibers

Equation (1) was solved by means of the Finite Element method using the Operator-Splitting method [19]. This technique reduces (1) to a nonlinear system of ODEs describing the cell action potential (reactive term), and a parabolic equation describing the potential diffusion among neighboring cells. An explicit Euler method with a time step of $0.02 \mathrm{msec}$ was used to solve the reactive term, while an Implicit Euler method was used for the diffusion equation. The tissue was divided in 980000 brick elements $400 \mu \mathrm{m}$ in size, for a total of 1013931 nodes. The fiber orientation was defined at the centroid of each element, and the anisotropic conductivity tensor calculated following the procedure described in [20]. Appropriate values for the conductivities were chosen to obtain a longitudinal conduction velocity (CV) of approximately $40 \mathrm{~cm} / \mathrm{sec}$ in the normal zone with an anisotropic velocity ratio of approximately 10:3.

A S1-S2 stimulation protocol was considered by delivering two planar pulses at the base of the slab defined by the yz plane (see Figure 1). The amplitude of the pulses was 1.5 times the diastolic threshold in the normal tissue, and had duration of $5 \mathrm{msec}$. The first stimulus (S1) was delivered at $t=75 \mathrm{msec}$ in order for the parameters in the model to reach steady state. The second stimulus (S2), of identical characteristics of S1, was delivered at the same location of S1 and at different intervals in order to determine the vulnerable window.

\section{Results and conclusions}

Figure 3 shows the membrane potential for the epicardial, endocardial, and mid planes of the slab for a fiber imbrication angle of $0^{\circ}$ across the wall. The figure also shows results for a 2D simulation with the same fiber orientation found in the mid plane of the slab. Pictures at $125 \mathrm{msec}$ and $250 \mathrm{msec}$ correspond to the membrane potential field within the basic stimulus (S1) interval. These pictures clearly demonstrate the effect of the fiber structure in the propagation pattern. However, for the mid plane, results show a similar depolarization pattern as for the $2 \mathrm{D}$ simulation. These observations are confirmed by the computed $\mathrm{CV}$ in the $\mathrm{NZ}, \mathrm{BZ}$, and $\mathrm{CZ}$ at the mid plane of the slab (see Table 1). In addition, cases with fiber imbrication angle varying from $0^{\circ}$ to: $2^{\circ}, 4^{\circ}, 6^{\circ}, 8^{\circ}$, and $10^{\circ}$, showed no appreciable changes in the CV. Regarding the vulnerable window $(\mathrm{VW})$, reentries were found for $\mathrm{S} 2$ in the range between 167-191 ms, leading to a vulnerable window of $25 \mathrm{msec}$, only a $4 \%$ larger than that found for the $2 \mathrm{D}$ case $(24 \mathrm{msec})$. The vulnerable window was not affected by the imbrication angle either. These results indicate that for an ischemic zone with a morphology given in Figure 1, the vulnerable window is not substantially affected by either the fiber structure or the 3D nature of the tissue, given the same results as for a 2D simulation. In fact, differences shown in Table 1 are most likely due to numerical errors during the computation of the CV.

\begin{tabular}{|l|c|c|c|c|}
\hline & \multicolumn{3}{|c|}{ Velocity [cm/sec] } & $\begin{array}{c}\text { Vulnerable } \\
\text { Window }\end{array}$ \\
\hline & NZ & BZ & CZ & \\
\hline 2D & 39.1 & 47.7 & 22.2 & $24 \mathrm{msec}$ \\
\hline 3D & 39.6 & 46.5 & 21.5 & $25 \mathrm{msec}$ \\
\hline
\end{tabular}

Table 1. Propagation velocity and vulnerable window for the $3 \mathrm{D}$ (with $0^{\circ}$ imbrication angle) and $2 \mathrm{D}$ cases

For the morphology of the acute ischemic region defined in Figure 1, the eight shaped reentry pattern was always found in the interior of the slab rather than in the epicardial surface (see Figure 3), where reentry appeared as an outcoming current. These findings are in agreement with experimental observations in dog hearts [4]. However, our investigations have also shown that eight shaped reentries are readily formed at the epicardial surface of the slab when the center of the ischemic region is moved toward this surface, a morphology change of the ischemic region. This change in the spatial location of the 
ischemic region not only affects the location of the reentry, but also the vulnerable window for the tissue. When a spherical ischemic zone with dimensions given in Figure 1 is centered in the epicardial surface of the slab, a $24 \%$ reduction in the vulnerable window with respect to values given in Table 1 are obtained. It not only indicates an important effect of the morphology of the ischemic zone on the vulnerable window, but also suggests that results obtained from $2 \mathrm{D}$ simulations may correspond to an upper bound for the vulnerable window.

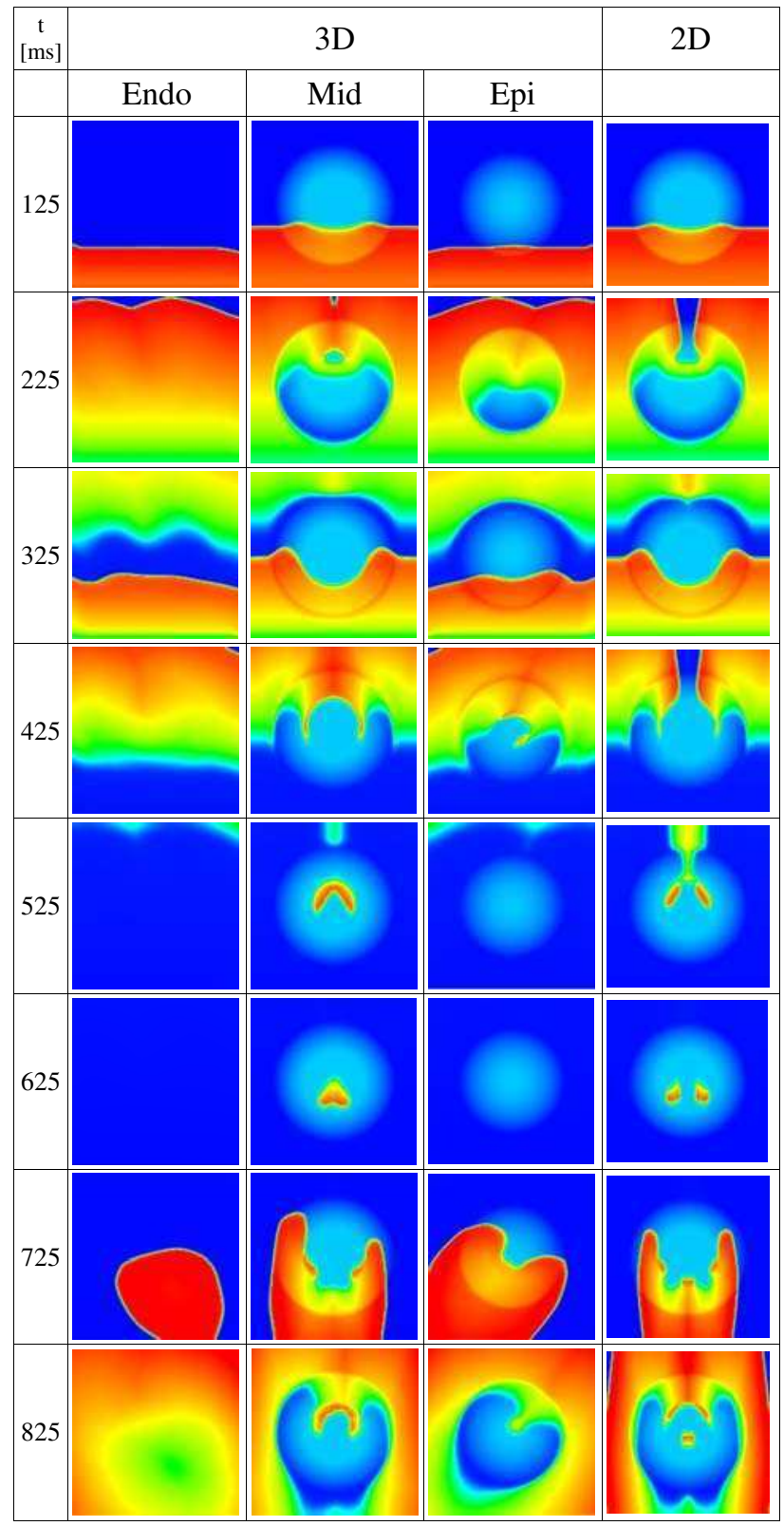

Figure 3. Transmembrane potential field at different times of the simulation. The tissue corresponds to a imbrication angle of $0^{\circ}$.

\section{Acknowledgements}

Aragon Government through the research project PIP113/2005.

\section{References}

[1] Rupert M., Zipes DP, Mechanics of sudden cardiac death. J. Clin. Invest. 2005; 115: 2305-2315.

[2] Carmeliet E, Cardiac Ionic Currents and Acute Ischemia: From Channels to Arrhythmias. Physiol. Rev. 1999; 79: 917-1017.

[3] Rodríguez B, Trayanova N, Noble D, Modeling Cardiac Ischemia. Ann. N.Y. Acad. Sci. 2006; 1080: 395-414.

[4] Janse, M. J., van Capelle, F. J. L., Morsink, H., Kleber, A. G., Wilms-Schopman, F., Cardinal, R., Naumann C., Durrer, D. Flow of 'injury current' and patterns of excitation during early ventricular arrhythmias in acute regional myocardial ischemia in isolated porcine and canine hearts. Circ. Res. 1981; 47: 151-165.

[5] Coronel R, Fiolet JW, Wilms-Schopman FJ, Schaapherder AF, Johnson TA, Gettes LS, Janse MJ. Distribution of extracellular potassium and its relation to electrophysiologic changes during acute myocardial ischemia in the isolated perfused porcine heart Circulation 1988; 77: 1125-1138.

[6] Coronel R. Heterogeneity in extracellular potassium concentration during early myocardial ischemia and reperfusion: implications for arrythmogenesis. Cardiovasc. Res. 1994; 28: 770-777.

[7] Janse MJ, Kleber AG, Electrophysiological changes and ventricular arrhythmias in the early phase of regional myocardial ischemia. Circ. Res. 1981; 49: 1069-1081.

[8] Ferrero JM, Trenor B, Rodríguez B, Saiz J. Electrical activity and reentry during acute myocardial ischemia: insights from simulations. Int. J. Bifurcat. Chaos 2003; 13(12): 3703-3715.

[9] Tice B, Rodríguez B, Trayanova N, Arrythmogenicity of transmural heterogeneities in a realistic model of regional ischemia. Heart Rhythm 2005; 2: S261.

[10] Rodríguez B, Tice B, Eason J, Aguel F, Trayanova N., Cardiac vulnerability to electric shocks during phase $1 \mathrm{~A}$ of acute global ischemia. Heart Rhythm 2004; 1(6): 695-793.

[11] Geselowitz D.B., Miller III, W.T., A bidomain model for anisotropic cardiac muscle. Ann. Biomed. Eng. 1983; 11:191-206.

[12] Luo CH, Rudy Y. A dynamic model of the cardiac ventricular action potential. I. Simulations of ionic currents and concentration changes. Circ. Res. 1994; 74: 1071-1096.

[13] Faber GM, Rudy Y. Action potential and contractility changes in $\left[\mathrm{Na}^{+}\right]_{\mathrm{i}}$ overloaded cardiac myocytes: a simulation study. Biophys J. 2000; 78: 2392-2404.

[14] Weiss JN, Shine KI. Effects of heart rate on extracellular [K+] accumulation during myocardial ischemia. Am. J. Physiol. 1986 250: H982-H991.

[15] Yatani A, Brown AM, Akaike N. Effect of extracellular $\mathrm{pH}$ on sodium current in isolated, single rat ventricular cells. J. Membr. Biol. 1984; 78: 163-168.

[16] Irisawa H, Sato R. Intra- and extracellular actions of proton on the calcium current of isolated guinea pig ventricular cells. Circ. Res. 1986; 59: 348-355.

[17] Weiss JN, Venkatesh N, Lamp ST. ATP-sensitive $\mathrm{K}^{+}$channels and cellular $\mathrm{K}^{+}$loss in hypoxic and ischemic mammalian ventricle. J. Physiol. (Lond.) 1992; 447: 649-673.

[18] Walfridson H, Odman S, Lund N. Myocardial oxygen pressure across the lateral border zone after acute coronary occlusion in the pig heart. Adv. Exp. Med. Biol. 1985; 191: 203-210.

[19] Qu Z, Garfinkel A., An Advanced Algorithm for Solving Partial Differential Equation in Cardiac Conduction. IEEE Trans. Biomed. Eng. 1999; 46(9):1166-1168.

[20] Sermesant, M, Delingette, H, Ayache, N. An electromechanical model of the heart for image analysis and simulation. IEEE Trans. Med. Imag. 2006; 25(5): 612-625. 